\title{
La obra poética de Marie de Romieu: ¿Traducción o imitación?
}

\author{
Manuela Álvarez Jurado \\ Universidad de Córdoba \\ ff1aljum@uco.es \\ https://dx.doi.org/10.12795/futhark.2011.i06.01
}

\begin{abstract}
The poetic work of Marie Romieu proves such an intricate maze of intertextual relations, that we are bound to find in her poems the traces left by her many readings of Classical, Italian and medieval French texts in addition to those of some contemporary authors. This sort of polyphony meets the poetess' attempt to rescue those texts from the readers' memory so that they can still linger throughout the ages to come.

By the use of a comparative analysis of several stanzas translated by Marie Romieu and her corresponding original texts we may draw the conclusion that Marie Romieu tries to imitate rather than translate them, and alongside with $\mathrm{J}$. du Bellay she considers translation as a kind of re-writing process, that is, a creative act wherefrom a new poetic work originates different from the source text where the 'l' of the translator has replaced that of the author with the purpose of adapting the work of a new cultural reality, in this case the French Renaissance.
\end{abstract}

Key Words:Translation and intertextuality, Adaptation, Imitation

Resumen : La obra poética de Marie de Romieu se pierde en un intrincado laberinto de relaciones intertextueales que nos permiten reconocer en sus poemas la huella dejada por las lecturas de textos clásicos, de la literatura italiana, de la literatura medieval así como de buena parte de autores contemporáneos a la autora. Esta polifonía responde al deseo de la poetisa de recuperar estos textos de la memoria del lector y salvaguardarlos del paso del tiempo. Gracias al análisis comparativo de una serie de estrofas de poemas traducidos por Marie de Romieu y losl textos originales, llegamos a la conclusión de que Marie de Romieu más que traducir imita y que la traducción es para ella, al igual que para su coétaneo, Joachim Du Bellay una reescritura, un acto de creación que produce una nueva obra, completamente diferente de la original y 
en la que el « yo » del traductor ha usurpado al « yo » del autor para finalmente adaptar su obra a una nueva realidad que no es otra que la del Renacimiento francés.

Palabras Clave : Traducción e intertextualidad, Adaptación, Imitación

El elevado número de traducciones que se llevaron a cabo en el Renacimiento ha hecho que éste sea considerado como "la edad de oro de la traducción". Este fenómeno iba unido a otros factores como el auge de la imprenta y el redescubrimiento de la Antigüedad. En Francia a todo esto se suma un creciente interés por las literaturas extranjeras contemporáneas como la italiana o la española principalmente. Este incremento del volumen de traducciones respondía a un deseo de apertura ya que en la Edad Media el campo de la traducción quedaba restringido a los textos consagrados por la Iglesia, es decir, el corpus de auctoritates. En el Renacimiento, por el contrario, el campo de lo "traducible" se amplia enormemente de tal modo que cualquier texto podía ser traducido sin importar su género ni la lengua en la que hubiese sido escrito e incluso se llevaron a cabo traducciones de traducciones, es decir, se tradujeron obras cuya lengua de origen era desconocida para el traductor. Todo esto llevó a la formación de un público asiduo lector de traducciones para el que leer significaba ante todo leer textos traducidos.

Por otra parte, se traducía sin seguir norma alguna, lo que llevó a Du Bellay a afirmar que la traducción (¿o más bien la traición?), era una tarea "inútil y perniciosa" ya que ésta tenía como resultado la profanación de los clásicos sin llegar a reportar al traductor la gloria deseada.

Ante esta situación, Étienne Dolet publica un breve pero valioso texto, La manière de bien écrire d'une langue en l'aultre(1540), en el que define los principios que debe respetar todo buen traductor ${ }^{1}$.

\footnotetext{
${ }^{1}$ Les règles fondamentales de la traduction:

1. En premier lieu il faut que le traducteur entende parfaitement le sens et matière de l'autheur qu'il traduict ; car par ceste intelligence il ne sera jamais obscur en
} 
Ante la probabilidad de que la traducción realizada no fuese del agrado del lector o de los críticos, los traductores tomaron la costumbre de excusarse ante estos por el trabajo llevado a cabo. Este tipo de intervención llegó a convertirse en un topos retórico: la captatio benevolentiae, que iba a su vez unido a una falta de reconocimiento del trabajo del traductor, otro de los topos más habituales del Renacimiento. Éste, más conocido como "la desgracia del traductor", no era sino un lugar común importado de Italia, según el cual la traducción era un "trabajo sin gloria" y la tarea del traductor era considerada más indigna que la del autor. El traductor tan sólo imitaba la obra que otro había creado, lo que hacía que la traducción no fuese considerada como

sa traduction et si l'autheur lequel il traduict est aucunement scabreux, il pourra rendre facile et du tout intelligible.

2. Que le traducteur ait perfaicte cognoissance de la langue de l'autheur qu'il traduict et soit pareillement excellent en la langue en la quelle il se mect à traduire : par ainsi il ne violera et n'amoidrira la majesté de l'une et l'autre langue.

3. II ne se fault pas asservir jusques à la qu'on rende mot pour mot. Chacusne langue a ses proprietés, translations en dictions, locutions, subtilités, vehemences a elle particulieres. Lesquelles, si le traducteur ignore, il fait tort à l'autheur qu'il traduit, et aussi à la langue en laquelle il le tourne. Je ne veulx taire icy la follie d'aucuns traducteurs lesquelz au lieu de liberté, se submettent à servitude. C'est assçavoir qu'ilz sont si sots qu'ilz s'efforcent de rendre ligne pour ligne ou vers pour vers. Par laquelle erreur ilz depravent souvent le sens de l'autheur qu'ilz traduisent et n'expriment la grace et perfection de l'une et l'autre langue.. Tu te garderas diligemment de ce vice qui ne demontre autre chose que l'ignorance du traducteur.

4. Le meilleur est de suivre le commun langage. S'il advient doncques que tu traduises quelque Livre Latin en ycelles il te faut garder d"usurper mots trop approchants du Latin et peu usité par le passé mais contente toy du commun. N'entends pas que je dye que le traducteur s'abstienne totallement de mots qui sont hors de l'usage commun car on sçait bien que la langue Grecque ou Latine est trop plus riche en dictions que la Françoyse. Qui nous contraint souvent d'user de mots pu fréquentés. Mais cela se doit faire à l'extreme necessité.

5. Rien aultre chose que l'observation des nombres oratoires: liaison et assemblement des dictions avec telle douceur que non selement l'ame s'en contente mais aussi les oreilles en sont ravies et en se faschent jamais d'une telle harmonie de langage. 
una labor creativa ya que al tratarse de una recreación, esto le restaba originalidad y por lo tanto calidad. ${ }^{2}$.

De este modo, el siglo XVI puede ser considerado como "el siglo de oro de la traducción" por diferentes motivos. En primer lugar porque es a partir de este momento cuando se empieza a utilizar el término "traducción" ya que en la Edad Media, el acto de traducir era designado por diferentes términos: translation (término que se ha conservado en inglés), expondré, turner, mettre en romanz, enromanchier, translater ${ }^{3}$ Esta diversidad de términos era debida a que en la Edad Media el acto de traducir no estaba desligado de otras prácticas como el comentario de textos, la recreación o la adaptación. ${ }^{4}$ Este fenómeno está íntimamente ligado a la diversidad de traducciones, adaptaciones, imitaciones y ¿por qué no? traiciones de los textos originales. En algunos casos nos encontramos con traducciones fieles y respetuosas con el texto original y, sin embargo, en otras ocasiones la traducción se aleja de éste a través de adaptaciones que hacen de ella un nuevo texto diferente de aquél que la había inspirado. En este caso hablamos de imitaciones o adaptaciones, o, como señalaron los poetas de La Pléiade, d'innutrition. Ya se trate de unas o de otras, todas responden al imperioso deseo renacentista de hacer prevalecer la herencia clásica que, de otro modo, hubiese caído en el olvido. Numerosos han sido y siguen siendo en la actualidad, los autores que frecuentan los textos clásicos o los provenientes de otras lenguas y otras culturas para hacer llegar a un público lector curioso y a la par deseoso de conocer, transmitir y conservar, los textos a los que no tenían acceso aquellos que no conocían la lengua en la que habían sido originariamente escritos.

\footnotetext{
2 SUSO LÓPEZ J , "Traductores, gramáticos y escritores en el siglo XVI en Francia", in Seis estudios sobre la traducción en los siglos XVI y XVII, Ed. Comares, Interlingua, Granada, 2003.

${ }^{3}$ SIMON, S, "Conflits de jurisdiction: la double signature du texte traduit", Meta: journal de traducteurs, Volumen 34, nำ2, Montréal, 1989.

4 "Au Moyen Âge (...) les frontières entre "sa" parole et celle "d'autrui" 'étaient fragiles. Équivoques, souvent tortueuses à dessein", BAKHTINE, M, Esthétique et théorie du roman, Paris, Gallimard, 1975, p.426.
}

Futhark 6 (2011)

Álvarez, La obra poética de Marie de

Romieu, 7-25

ISSN 1886-9300 
El siglo XVI se sitúa en una etapa histórica de la traducción cuya reflexión teórica está marcada por el precepto de Cicerón de "no traducir verbum, pro verbo" 5 . La práctica de la traducción ad sensum a finales del siglo XV acompañada, en la mayoría de los casos, de glosarios o comentarios parafrásticos, prevaleció a lo largo de la primera mitad del siglo XVI en Francia. Sin embargo, la segunda mitad del siglo XVI se verá fundamentalmente dominada por la traducción literal o traducción ad verbum.

En el Renacimiento las traducciones se engloban dentro de un género mucho más amplio que es la “imitación”. Esta clasificación proviene de la división en categorías preconizada por Quintiliano: la traducción fiel, la paráfrasis y el tratamiento libre del texto fuente. ${ }^{6}$ En esta época cualquier texto se consideraba imitación de otros textos, anteriores o contemporáneos. Al ser imitados, estos textos podían ser simplemente igualados en cuanto a calidad o incluso superados por su traducción. ${ }^{7}$ De este modo, imitar consistía en hacer una obra original apropiándose de los temas y formas de las obras imitadas. A lo largo del siglo XVI, la traducción fue considerada por algunos autores como la forma suprema de la imitación, así para Peletier du Mans la plus vraie espèce d'imitation c'est de traduire. Car imiter n'est autre chose que vouloir faire ce que fait autre: ainsi que fait le Traducteur. ${ }^{8}$. En cuanto a la traducción poética, las opiniones son muy diversas, así Thomas Sébillet consideraba que la version ou traduction est aujourd'hui le poème le plus fréquent et mieux reçu des estimés Poetes et des doctes lecteurs ${ }^{9}$. Du Bellay, sin embargo, en su Défense et Illustration de la langue française, recomienda no traducir a los poetas. Tanto para Du Bellay como para el resto de los poetas de la Pléiade, la traducción poética no era considerada como una obra creativa:

${ }^{5}$ STEINER (G) 1991, Après Babel: une poétique du dire et de la traduction, Albin Michel, Paris, pp.225-226.

${ }^{6}$ QUINTILIANO, Institutio oratoria, $X, 5,2-11$.

${ }^{7}$ "ll faut que nos traductions, pour être parfaites, paraissent comme d'autres originaux, et comme une nouvelle production; et qu'elles fassent demander aux lecteurs si les ouvrages qu'on a traduits sont aussi beaux que ces traductions ", Antoine le Maistre, Les sermons de S. Bernard, 1658

${ }^{8}$ PELETIER DU MANS (J) 1971, Art Poétique (1555), Slatkine Reprints, Ginebra.

${ }^{9}$ SEBILLET, (Th) 1972, Art Poëtique françoys (1549), Slatkine Reprints, Ginebra. 
Mais que dirais-je d'aucuns, vraiment mieux dignes d'être appelés traditeurs que traducteurs? (...) Celui donc qui voudra faire œuvre digne de prix en son vulgaire, laisse ce labeur de traduire, principalement les poètes, à ceux qui, de chose laborieuse et peu profitable, j'ose dire encore inutile, voire pernicieuse à l'accroissement de leur langue, emportent à bon droit plus de molestie que de gloire. ${ }^{10}$

En el Renacimiento, por tanto, se condena la traducción literal pero se considera que para traducir bien no sólo había que transferir fielmente el sentido del texto original sino que el texto traducido tenía que ser apropiado desde un punto de vista estilístico. ${ }^{11}$ De este modo, el concepto de traducción en el siglo XVI va unido a un compromiso con la estética del texto. No en vano, las artes poéticas renacentistas dedicaron sus páginas al estudio de la traducción, considerándola como un género literario.

En 1581 apareció en París la primera obra de la poetisa francesa Marie de Romieu: Les Premieres oeuvres poétiques de Ma Damoiselle Marie de Romieu Vivarois ${ }^{12}$. Ésta fue reeditada en 1878 en tirada limitada y con prefacio y notas de Prosper Blanchemain mientras que el resto de la obra de la autora apareció publicada en el volumen de poemas de su hermano, el poeta Jacques de Romieu ${ }^{13}$.

De Marie de Romieu no existen sino incompletas noticias bibliográficas, por las que se sabe que perteneció a una noble familia del Vivarais, quizá de Viviers. Otros sin embargo consideran que la poetisa era hija de un modesto panadero. Probablemente estaba casada ya que, según palabras de la propia autora, debido a la dedicación que requería su hogar, no pudo consagrar todo el tiempo que hubiera deseado a su pasión por las letras:

${ }^{10}$ DU BELLAY, J. (1549), La défense et illustration de la langue française, Livre I, chap. 6 , «Des mauvais traducteurs, et de ne traduire les poètes »

11 Ibidem, p. 67.

12 DE ROMIEU (M) 1970, Premières oeuvres poétiques, éd. André Winandy, Droz, Ginebra.

${ }^{13}$ La publicación más reciente data de 1972 con estudio y edición crítica de André Winandy, aunque debo señalar que se encuentra en prensa la reedición de las obras completas de la autora en Honoré Champion a cargo del profesor de Literatura Francesa de la Universidad de Québec, Claude La Charité.

Futhark 6 (2011)

Álvarez, La obra poética de Marie de

Romieu, 7-25

ISSN 1886-9300 
Prenez donc en bonne part, mon Frere, ce mien brief discours de nostre mesnage de vacquer comme vous dedié aux Muses à chose si belle et divine que les vers ${ }^{14}$

Un primer acercamiento a sus composiciones nos permite aventurar la sólida formación intelectual de la autora. La obra poética de Marie de Romieu se pierde en un intrincado laberinto de relaciones intertextuales; en sus poemas reconocemos las huellas dejadas por sus lecturas: autores clásicos como Virgilio, Anacreonte, Ovidio o Hesíodo, italianos como Sannazaro, Petrarca o Landi o autores franceses medievales como Christine de Pisan y contemporáneos de la autora como Desportes, Estienne e incluso el propio Ronsard. En el epigrama que encabeza la obra de Marie de Romieu, su hermano y editor, el poeta Jacques de Romieu, la presenta como una docta femina:

\footnotetext{
Si sopor hic (sacris edocta sororibus esset, Offerret pedibus carmina plura tuis,

Sat novi; sed abest. At quaedam carmina fratri

Accipe, quicquid id est, laeta, nova Margari, fronte, Accipe foemineos, foemina facta, modos. ${ }^{15}$
}

El hecho de que Marie de Romieu se dedicara a la traducción quiere decir que no sólo conocía el latín sino que probablemente también conoció alguna otra lengua tan profundamente como para emprender la traducción de varias obras, con lo que rivalizaría con autores ya consagrados que habían realizado estas traducciones anteriormente. Aprender latín, acceder a una cultura lingüística, filosófica, lógica, representaba para las mujeres de la época una empresa casi imposible. Al igual que Marie de Romieu, numerosas personas debieron aprender los rudimentos del latín o de otras lenguas mediante la confrontación de los textos traducidos con el original o bien

14 "Epistre a mon frere", Les premieres oeuvres poétiques, éd. André Winandy, Genève, Droz, 1972, p.p. 4-5.

${ }^{15}$ Op. Cit., p. 3. 
de modo autodidáctico ${ }^{16}$. El hecho de que una mujer "osara traducir obras literarias del latín al francés" constituía de por sí una auténtica revolución para la época, de tal modo que numerosos eruditos vieron tal iniciativa como una intromisión de las mujeres en un terreno prohibido, reservado a los hombres"17.

Por este motivo, Marie de Romieu, al igual que muchas escritoras de su época fue objeto de las más duras críticas por su labor traductora:

Son livre finit par une complainte funèbre sur la mort de nostre sauveur Jésus-Christ, complainte qui non plus que toutes ses autres pièces n'est pas de son invention mais de celle de mon fameux Sannazar qui l'avoit faite en beaux vers latins que j'ay aussi traduite autreffois, auparavant d'avoir veu cette ancienne version: et j'ay mesme rendu a l'autheur l'honneur qu'il méritoit, ce que n'a point fait cette noble damoiselle qui emprunte franchement sans croire estre obligée de rendre; puisqu'il n'y a pas un titre de ses Poèmes qui ne marque que ce soit imitation ou traduction de quelque autheur ancien ou moderne. ${ }^{18}$

Numerosos son los poemas que Marie de Romieu tradujo del italiano lo que no quiere decir que tuviese conocimiento de esta lengua ya que pudo haberse servido de las múltiples traducciones francesas o latinas que circulaban de estas obras. Le "Brief discours que l'excellence de la femme surpasse celle de l'homme ", retoma la célebre querella medieval en la que participaron entre otros Christine de Pisan o Jean de Meung. La composición de Marie de Romieu es una indiscutible imitación de Paradoxes de Charles Estienne que, a su vez, es una traducción de Paradossi del escritor italiano Ortensio Landi. De este

${ }^{16}$ FERNANDEZ FRAILE, (M.E) 2003 , "Mujer y traducción en Francia en el siglo XVII" in Seis estudios sobre la traducción en los siglos XVI y XVII, Ed. Comares, Interlingua, Granada.

${ }^{17}$ DEZON-JONES,( E.)1988, Fragments d'un discours féminin, Paris, José Corti, pp. 4849.

${ }^{18}$ COLLETET, (G), 1876, L'Histoire des Poètes François, citado por Henry Vaschalde en Histoire des Poètes du Vivarais, París, Auguste Aubray, p- 47.

Futhark 6 (2011)

Álvarez, La obra poética de Marie de

Romieu, 7-25

ISSN 1886-9300 
modo resulta difícil determinar si Marie de Romieu consultó también el texto original italiano o solo accedió a la traducción francesa.

En la traducción de Estienne podemos observar algunas adaptaciones como la sustitución de la enumeración de damas italianas de Landi por un catálogo de mujeres francesas de la época. Del mismo modo, Marie de Romieu, como conclusión a su "Brief discours", en uno de los pocos pasajes de su obra que no son traducción o adaptación, reúne a diez mujeres célebres del Renacimiento francés:

Vien donc soeur des neuf soeurs, et quatrieme Charite,

Ma contesse de Retz, vien que tu sois escrite

La premiere en mes vers : le Grec t'est familier,

De ta bouche ressort un parler singulier,

Qui contente les Rois et leur Cour magnifique,

Le latin t'est commun, et la langue Italique.(...)

Venez apres Morel, Charamont, Elisenes,

Des Roches de Poictiers Graces Pieriennes.

Vous aussi qui tenez le sceptre Navarrois (...),

La perle de Vallois qui est au Ciel Deesse

Maintenant pour jamais. Toy qui regis icy

La France qui se rend à ta douce mercy

Voy ce qu'en ta faveur grand Royne Catherine

J'ecris pour haut tonner la race feminine. ${ }^{19}$

En el caso de Marie de Romieu, volvemos a encontrar los mismos argumentos, los exempla, los calcos verbales y léxicos así como las estructuras sintácticas de Estienne:

19 DE ROMIEU, M. Op.cit. pp.20-21. 
Que iugerez vous de cueur de ceste noble dame, qui daigna recevoir honorablement en ses terres tout l'ost des Romains, avec une liberalité si grande, que la memoire en dure encore pour le iourdhuy ?20

Lisez le fait hautain de ceste noble Dame,

De qui pour tout jamais courra cy bas la Fame,

Qui daigna recevoir d'une honorable main.

Liberalle sans plus, tout le grand ost Romain. ${ }^{21}$

Qui est pour vous montrer, que tout ainsi d'elles naissent les hommes, aussi font les sciences que nous appelons humaines. ${ }^{22}$

Qui est pour vous montrer que, comme d'elles naissent Les hommes, et encore par leur moyen accroissent, Les sciences aussi qu'on dit d'humanité (...) ${ }^{23}$

Sapho trouua les vers qui de son nom furent appelez Sapphicques. ${ }^{24}$

(...)Saphe trouva les vers

Qui depuis, de son nom, furent nommez Saphiques ${ }^{25}$

El poema XXXII de Marie de Romieu «Imitation d'un sonnet de Petrarque " se añade a la larga lista de traducciones e imitaciones del célebre soneto del poeta italiano Pace non trovo ${ }^{26}$, poema número CXXXIV de su Canzionere.

Pace non trovo e non ho da far guerra

E temo, e spero; e ardo e sono un ghiaccio;

20 ESTIENNE, Ch., Paradoxes, París, 1553

${ }^{21}$ DE ROMIEU, M. Op. cit., p. 15.

${ }^{22}$ ESTIENNE, Ch, Op.cit.

${ }^{23}$ DE ROMIEU, M. Op. cit. p.19.

${ }^{24}$ ESTIENNE, Ch. Op. cit.

25 DE ROMIEU, M. Op, cit.,p. 19.

${ }^{26}$ Entre otros autores este poema inspiró a Louise Labé, Ronsard o Peletier du Mans.

Futhark 6 (2011)

Álvarez, La obra poética de Marie de

Romieu, 7-25

ISSN 1886-9300 
E volo sopra'l cielo, e giaccio in terra;

E nulla stringo, e tutto il mondo abbraccio.

Tal m'ha in pregion, che non m'apre nè sera,

Nè per suo mi riten nè scioglie il laccio;

E non m'ancide Amore, e non mi sferra,

Nè mi vuol vivo, nè mi trae d'impaccio.

Veggio senz'occhi, e non ho lengua, e grido;

E bramo di perire, e chieggio aita;

$E$ ho in odio me stesso, e amo altrui.

Pascomi di dolor piangendo rido;

Egualmente mi spiace morte e vita:

In questo statu son, donna, per voi.

Veamos a continuación el poema de Marie de Romieu:

Paix je ne treuve, et ne puis faire guerre ;

J'espere et crains ; je brusle et je suis glace;

Rien je n'estrains, et tout ce rond j'embrasse ;

Je vole au ciel, et si je suis en terre.

Je suis captif, et si rien ne m'enserre;

Enrethé suis, et rien ne m'entre-lace;

Rien je ne veux, et si j'aime la face

De celle là sur toutes qui m'enferre.

Je voy sans yeux, sans cœur me convient vivre ;

Le dueil me paist, le plaisir me veut suivre ;

Je veux perir, l'aide le cœur m'enflamme ;

Autruy me plaist, et moy mesme m'ennuye ;

Egalement veux la mort et la vie :

En tel estat je suis pour vous, ma-dame ${ }^{27}$.

En este caso, Claude La Charitée ${ }^{28}$ manifiesta que sin lugar a dudas, la poetisa ha accedido al texto original italiano. Aunque Marie de

${ }^{27}$ DE ROMIEU, M., Op. cit., pp. 104.

28 LA CHARITÉ (Cl.) 2000, " Le problème de l'attribution de l'instruction pour les jeunes dames (1572)", in Bibliothèque d'Humanisme et Renaissance, Tome LXII, no1, pp.125-126. 
Romieu no traduce el poema, sino que realiza una imitación de éste a través del calco de la estructura rítmica de los dos últimos versos, al contrario de lo que habían hecho los otros imitadores y traductores.

La «Complainte de la mort de nostre Saveur Jesus Christ » es una imitación directa de la «Lamentatio de morte Christi Domini » de Sannazaro. En este caso, la poetisa francesa imitó sin lugar a dudas el poema neolatino original. La autora abandona la imitación servil para llevar a cabo una recreación del poema italiano, dando lugar a un poema creativo, original y de gran emotividad en el que la poetisa vierte sus propios sentimientos. Marie de Romieu, siguiendo la tradición humanista, mezcla en su poema elementos paganos y elementos cristianos aunque su intención sea el hacerse portavoz de la ortodoxia cristiana. Por otra parte, la considerable diferencia de extensión de estas dos composiciones (mientras que la de Sannazaro consta de 46 versos, la de Marie de Romieu se extiende hasta 188) es otra prueba más de la intención de la poetisa francesa de recrear el poema italiano vertiéndolo al francés y creando un poema completamente diferente.

Marie de Romieu tradujo igualmente algunas obras latinas de escritores franceses contemporáneos. El poema XII es una transposición del Nihil de Jean Passerat. En este caso no se trata, al igual que en la mayor parte de sus traducciones, de una traducción exacta, literal, sino que Marie de Romieu adapta el texto latino a la estructura propia de la lengua francesa:

Mun best, festis quod possim aferre Calendis, Sccine Castalius nobis exaruit humor? Usque adeo ingenij nostri est exhausta facultas, Immunem ut videat redeuntes janitor anni? ${ }^{29}$

Voyci le bon Janus qui vient avec sa bande Pour celebrer le jour de sa sainte Calende : Mais coustumier ment il veut un don gaillard, Et je n'ay pour offrir don a ce bon vieillard. Est-ce ainsi que l'humeur qui desceend de Permesse ${ }^{30}$

29 PASSERAT, J., Nihil per lonannem Passeratium, París, 1588.

${ }^{30}$ DE ROMIEU, M., Op.cit., p. 75.

Futhark 6 (2011)

Álvarez, La obra poética de Marie de

Romieu, 7-25

ISSN 1886-9300 
Por otra parte la "Traduction du Latin de M. de la Val".de Marie de Romieu es un calco fiel del poema que Joachim de la Val dedicó a Jacques de Romieu. A continuación se presentan ambos poemas:

\author{
Si quis molliculos concinné spirat amores \\ Et canit Heroas, bella, virumque, Deos. \\ Dicite, Pierides, Romaeus, natus olympi \\ Viuarienei gloria sola, soli : \\ Vnus erit veridi cinget qui Temporor fronde, \\ Praemia virtutis praemia digna suae. \\ Quem cupiunt igitur Charites, doctaeque Puellas, \\ Complet \& Aonij fontis vterque sinus. \\ Gallia diues opum ne dedigneris alumnum : \\ Magna sed in iuuenis corde latere puta. ${ }^{31}$ I
}

Si quelcun proprement soupire amours nouveaux Et chante les Heros, les dieux, l'homme, la guerre, Dites, Muses, ces mots : "De Romieu, de sa terre Seul los, \& nourriçon du ciel porte flambeaux,

Un entre tous sera à qui la verte feuille,

De la vertu le prix, son docte front ceindra ;

Qui puis apres par tout estimer le fera

Quoy qu'envie ou le temps opinatrer s'y veuille. ”

Donc, France, riche en biens, ne dedaigne un filz cher.

Que les Charites Sœurs, \& les doctes pucelles

Veuillent, bonnes tenir sans cesser aupres d'elles ;

Ains dans un ieune cœur croi maint Grand se cacher. ${ }^{32}$

Como ya se ha señalado anteriormente, la extensión de las traducciones de Marie de Romieu superan la del original. Esta circunstancia, en principio, viene determinada por la propia naturaleza de la traducción. Al ser ésta considerada como reescritura, tiende hacia

${ }^{31}$ DE ROMIEU, J., Mélanges.

32 DE ROMIEU, M., Op. cit, p. 128. 
una mayor extensión de la que la autora se sirve para añadir algunos elementos nuevos que enriquecen y completan el poema.

$\mathrm{Al}$ analizar las diferentes traducciones de Marie de Romieu, se puede constatar que no nos encontramos en el terreno de la traducción propiamente dicha sino que más bien se trata de una auténtica labor de imitación, en el que la poetisa lleva a cabo una adaptación de los textos originales al gusto de su época con el fin, sin lugar a dudas, de facilitar la comprensión lectora y hacerlos más cercanos a sus coetáneos. Tanto los poemas originales como las traducciones se presentan como dos poemas distintos. Al fin y al cabo, todo poema es en sí mismo una reescritura y traducción de otro que, a su vez, lo es de otro estableciéndose así un juego literario donde se pierden el original y la autoría. La obra poética de Marie de Romieu se presenta como un complejo e intrincado laberinto polifónico en el que podemos oir las voces de los diferentes textos que la autora adaptó e imitó.

\section{REFERENCIAS BIBLIOGRÁFICAS:}

BAKHTINE, (M.) 1975, Esthétique et théorie du roman, Paris, Gallimard. 
DE ROMIEU, (M.) 1972, Les premieres oeuvres poétiques, éd. André Winandy, Genève, Droz.

DEZON-JONES, (E.) 1988, Fragments d'un discours féminin, París, José Corti, pp. 48-49.

ESTIENNE, (Ch.) 1553, Paradoxes, París.

FERNÁNDEZ FRAILE, (M.E.) 2003, « Mujer y traducción en Francia en el siglo XVII", in Seis estudios sobre la traducción en los siglos XVI y XVII, ed. Comares Interlingua, Granada.

LA CHARITÉ (Cl.) 2000, “ Le problème de l'attribution de l'instruction pour les jeunes dames (1572)", in Bibliothèque d'Humanisme et Renaissance, Tome LXII, №1.

LE MAISTRE, (A.) 1658, Les sermons de S. Bernard.

PASSERAT, (J.) 1588, Nihil per lonannem Passeratium, París.

PELETIER DU MANS (J.) 1971, Art Poétique (1555), Slatkine Reprints, Ginebra

SIMON, (S.), 1989, "Conflits de jurisdiction: la double signature du texte traduit", Meta: journal de traducteurs, Volumen 34, №2, Montréal.

SEBILLET, (Th.) 1972 Art Poëtique françoys (1549), Slatkine Reprints, Ginebra.

STEINER (G.) 1991, Après Babel: une poétique du dire et de la traduction, Albin Michel, Paris.

SUSO LÓPEZ (J.) 2003, "Traductores, gramáticos y escritores en el siglo XVI en Francia", in Seis estudios sobre la traducción en los siglos XVI y XVII, Ed. Comares, Interlingua, Granada. 